\title{
Pumping of Vibrational Excitations in the Coulomb-Blockade Regime in a Suspended Carbon Nanotube
}

\author{
A. K. Hüttel, ${ }^{1, *}$ B. Witkamp, ${ }^{1}$ M. Leijnse, ${ }^{2,3,4}$ M. R. Wegewijs, ${ }^{2,3,4}$ and H. S. J. van der Zant ${ }^{1}$ \\ ${ }^{1}$ Molecular Electronics and Devices, Kavli Institute of Nanoscience, Delft University of Technology, \\ PO Box 5046, 2600 GA Delft, The Netherlands \\ ${ }^{2}$ Institut für Theoretische Physik A, RWTH Aachen, 52056 Aachen, Germany \\ ${ }^{3}$ Institut für Festkörper-Forschung-Theorie 3, Forschungszentrum Jülich, 52425 Jülich, Germany \\ ${ }^{4}$ Jülich Aachen Research Alliance-Fundamentals of Future Information Technology (JARA-FIT)
}

(Received 9 December 2008; published 2 June 2009)

\begin{abstract}
Low-temperature transport spectroscopy measurements on a suspended few-hole carbon nanotube quantum dot are presented, showing a gate-dependent harmonic excitation spectrum which, strikingly, occurs in the Coulomb-blockade regime. The quantized excitation energy corresponds to the scale expected for longitudinal vibrations of the nanotube. The electronic transport processes are identified as cotunnel-assisted sequential tunneling, resulting from nonequilibrium occupation of the mechanical mode. They appear only above a high-bias threshold at the scale of electronic nanotube excitations. We discuss models for the pumping process that explain the enhancement of the nonequilibrium occupation and show that it is connected to a subtle interplay between electronic and vibrational degrees of freedom.
\end{abstract}

DOI: 10.1103/PhysRevLett.102.225501

PACS numbers: 63.22.Gh, 73.23.Hk, 73.63.Fg

The coupling of vibrational modes and electronic transport in nanoscale systems and, in particular, quantum dots is currently the focus of many theoretical [1,2] and experimental [3-9] research efforts. In this respect, single wall carbon nanotubes (SWCNTs) provide a unique mesoscopic system where both bulk beam mechanics $[3,4]$ and quantization of phonon modes $[5,6,9]$ have already been demonstrated. Low-temperature Coulomb-blockade (CB) spectroscopy on quantum dots formed within the nanotube $[10,11]$ has led to a well-developed understanding of the electronic structure of SWCNTs [12-14]. In suspended SWCNT quantum dots, transport spectroscopy has also revealed the Franck-Condon effect [1], where the quantized vibrations of the nanotube become visible in single electron tunneling (SET) at finite bias. A large electron phonon coupling [6,9] and first indications of vibrational phenomena in cotunneling have been observed $[9,15]$.

In this Letter we present low-temperature transport measurements on a suspended CNT quantum dot system. We observe signatures of nonequilibrium population of the quantized mechanical oscillations in the transport spectrum, revealed by cotunnel-assisted sequential electron tunneling (COSET). The nonequilibrium occupation is enhanced ("pumped") by higher-order tunnel processes. Detailed models are discussed which explain the observations.

The basic device geometry is sketched in Fig. 1(a). A highly $p^{++}$doped silicon wafer, also functioning as backgate, with $500 \mathrm{~nm}$ thermally grown oxide on top provides the starting point of the sample preparation. After lithographic fabrication of marker structures and localized deposition of growth catalyst [16], carbon nanotubes are grown in situ by chemical vapor deposition and located using atomic force microscopy. Contact electrodes consist- ing of $5 \mathrm{~nm}$ chromium and $50 \mathrm{~nm}$ gold are deposited on top of the nanotubes. In a last step, the nanotube devices are suspended using wet etching in buffered hydrofluoric acid.

Measurements were performed in a dilution refrigerator with a base temperature $T_{\mathrm{MC}} \lesssim 20 \mathrm{mK}$ and an electron temperature $T_{\mathrm{el}} \simeq 100 \mathrm{mK}$. Figure 1(b) shows the differential conductance $d I / d V_{\mathrm{SD}}$ as function of backgate (substrate) voltage $V_{g}$ and bias voltage $V_{\mathrm{SD}}$ of one particular suspended nanotube quantum dot. This sample has a lithographically designed length of $l=250 \mathrm{~nm}$ and was-from transport measurements as in Fig. 1(b)—identified to be a semiconducting nanotube with a band gap of $E_{g}=$ $200 \mathrm{meV}$. Band gap energy and device length lead to a predicted energy scale of $\Delta E \simeq 6 \mathrm{meV}$ of orbital electronic excitations [14]. In the measurement of Fig. 1(b), the basic structure of diamond-shaped CB regions with a

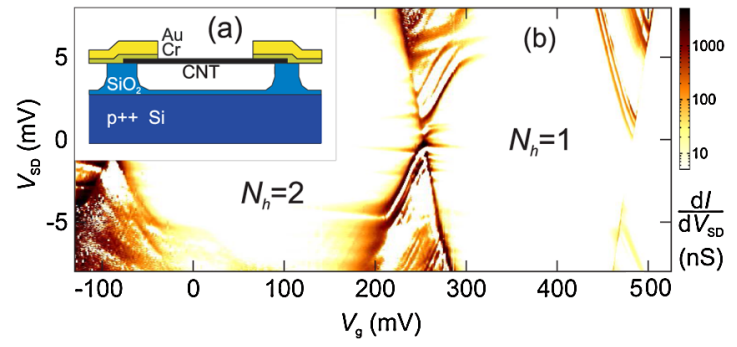

FIG. 1 (color online). (a) Side-view drawing of the sample geometry. (b) Overview measurement of the differential conductance $d I / d V_{\mathrm{SD}}$ of the nanotube quantum dot system in the few-hole region $\left(1 \leq N_{h} \leq 2\right.$, with $N_{h}=0$ and $N_{h}=3$ visible at the edges of the plot) as function of backgate voltage $V_{g}$ and bias voltage $V_{\mathrm{SD}}$ (logarithmic color scale, negative differential conductance is plotted white). In the $\mathrm{CB}$ regions, the number of trapped valence band holes $N_{h}$ is indicated. 
fixed trapped charge, as expected for a single quantum dot, is clearly visible. Since the band gap position is known, we can identify the charge states as $N_{h}=1$ and $N_{h}=2$, respectively.

Figure 2(a) displays a detail measurement of the single electron tunneling region with $1 \leq N_{h} \leq 2$ at low negative bias, and the adjacent $N_{h}=2 \mathrm{CB}$ region. A rich spectrum of equidistant excitation lines with positive slope, corresponding to excitations of the $N_{h}=1$ system (see, e.g., Ref. [14]), is found in SET [17]. This is also detailed in the enlarged plot of Fig. 2(b) (see arrows). Figure 2(c) shows the corresponding excitation energies as function of line number [18]. We assign these excitations to a harmonic vibration whose frequency is $\hbar \omega=0.425 \pm 0.004 \mathrm{meV}$, in good agreement with the bulk mechanics prediction of $\hbar \omega_{\text {vib }}=0.44 \mathrm{meV}$ for the longitudinal vibration mode of a $250 \mathrm{~nm}$ long nanotube segment [6]. In addition, the data of Figs. 2(a) and 2(b) reveal three faint excitation lines with
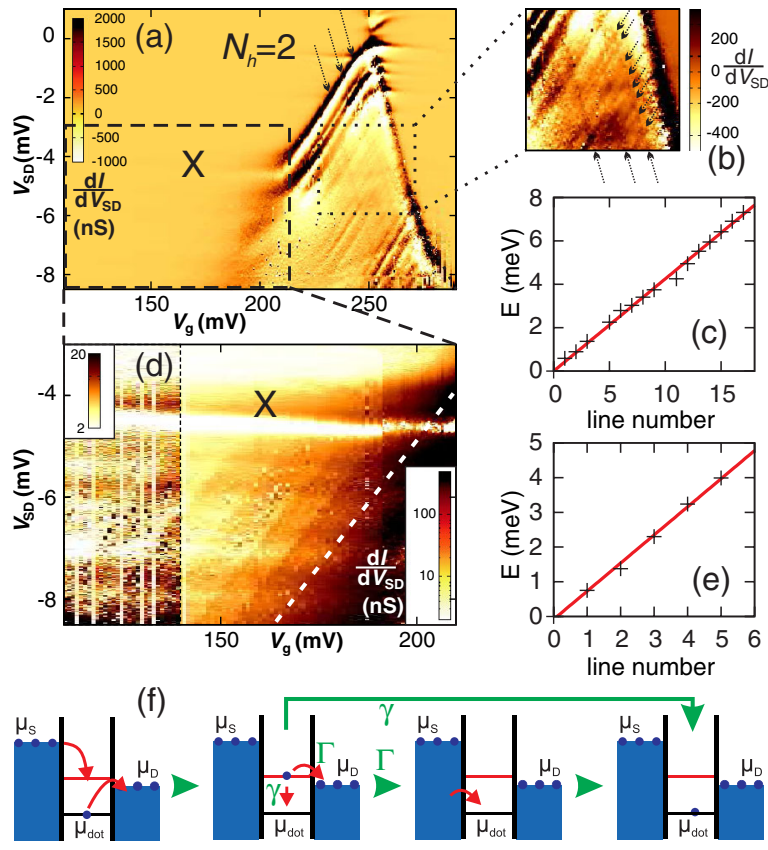

FIG. 2 (color online). (a) Differential conductance $d I / d V_{\mathrm{SD}}$ in the region where the nanotube is charged with $1 \leq N_{h} \leq 2$ holes, as function of gate voltage $V_{g}$ and bias voltage $V_{\mathrm{SD}}$ (linear color scale). (b) Detail zoom of part of the SET region with $1 \leq$ $N_{h} \leq 2$, as marked in (a), using a different color scale. In both (a) and (b), arrows point out line features corresponding to excited states (see text) [17]. (c) Excitation energies corresponding to the lines of enhanced $d I / d V_{\mathrm{SD}}$ in (a) and (b) with positive slope; the $x$ axis is the line number. The solid line is a linear fit, resulting in an average energy difference of $\Delta E=$ $0.425 \pm 0.004 \mathrm{meV}$ per line. (d) Detail zoom of the $\mathrm{CB}$ region with $N_{h}=2$, as marked in (a) (logarithmic color scale) [17]. A white dashed line sketches the edge of the SET region. For better contrast, different color scale ranges are chosen in parts of the plot. (e) Relative excitation energies for the line features in (d), using the same parameters for position to energy conversion. A linear fit gives $\Delta E=0.810 \pm 0.025 \mathrm{meV}$. (f) Schematic of cotunnel-assisted sequential tunneling (COSET) (see text). negative slope (i.e., $N_{h}=2$ excitations), marked by three black arrows and separated by approximately $0.7 \mathrm{meV}$. It is difficult to confirm a harmonic spectrum because of the faintness of the three lines.

Figure 2(d) enlarges the region outlined in Fig. 2(a) by a black dashed rectangle, plotting the differential conductance in logarithmic color scale. Here, CB stabilizes a total charge of $N_{h}=2$ holes on the nanotube, suppressing SET. Surprisingly, a pattern of gate-dependent excitation lines in the $\mathrm{CB}$ region, parallel to the edge of the SET region, emerges [17]. Their relative excitation energies are plotted in Fig. 2(e). A regular spacing corresponding to a harmonic oscillator energy of $\hbar \omega=0.810 \pm 0.025 \mathrm{meV}$ is visible, close to the above-mentioned energy scale $0.7 \mathrm{meV}$ of the $N_{h}=2$ excitations in SET.

The most straightforward explanation for these lines is that they correspond to COSET processes [19]. These are multistep processes, as sketched in Fig. 2(f). Inelastic cotunneling leaves a quantum dot in an excited state (see second panel in the figure), which is possible at any voltage above the energy of this state. Subsequently, sequential tunneling to the drain electrode can follow for voltages close to the Coulomb diamond edges, even if the Coulombblockaded ground state does not allow it. For this to happen, the rate $\Gamma$ for tunneling out has to be comparable to or larger than the relaxation rate $\gamma$ to the ground state.

In general, COSET processes can involve intrinsic (e.g., orbital) excitations of quantum dots as well as vibrational states. Here, as opposed to the measurements of Ref. [19], the highly regular excitation spectrum observed in Fig. 2(d) strongly indicates a vibrational origin. The vibrational COSET lines are related to the two-hole charge state vibrational excitations seen in SET with negative slope. As the vibrational energy exceeds thermal broadening $\left(\hbar \omega \gg k_{B} T\right)$, the observation of multiple excitations in CB strongly suggests storage and subsequent release of energy in the vibrational mode, involving phonon absorption sidebands $[2,5,20]$. This stands in contrast to the usual assumption that vibrational relaxation is fast and that the mechanical system is predominantly found in its ground state. The required energy is provided by inelastic cotunneling, exciting the vibrational mode and causing its nonequilibrium occupation.

A particularly interesting feature of the measurement of Figs. 2(a)-2(e) is that the COSET lines can only be observed beyond a weakly gate-dependent threshold at $V_{\mathrm{SD}} \simeq$ $-4 \mathrm{mV}$ (marked $X$ in the plots), where the differential conductance is enhanced; i.e., they only occur at energies higher than $4 \mathrm{meV}$. The line feature marked with $X$ in the measurements represents the onset of an inelastic cotunneling current, corresponding to an electronic excitation of the quantum dot. To understand the resulting interplay between mechanical and electronic excitations we have performed model calculations, illustrated in Fig. 3. The transport calculations account for strong Coulomb effects as well as tunneling processes up to fourth order in the tunneling Hamiltonian, responsible for cotunneling, line 
broadening and level renormalization which are important close to SET resonance; see Ref. [20] for details.

The two-hole ground state and its vibrational excitations are the origin of COSET lines as observed in the experiment. To demonstrate this idea, it suffices to consider a model with several equidistant states $A-E$ [see Fig. 3(a), panel (1), and also [17]], which are coupled with the same rate $\Gamma$ to the one-hole ground state. In addition, an excited two-hole state $X$ supporting a current with rate $\Gamma^{\prime}$ is introduced. Since the edge of the CB diamond becomes very broad in the experiment beyond this excitation, an estimate of the ratio of $\Gamma$ and $\Gamma^{\prime}$ based on the SET current is difficult. However, the strong signature of the inelastic cotunneling line associated with state $X$ indicates that $\Gamma^{\prime} \gg \Gamma$. The result of a calculation based on this model is shown in Fig. 3(b). The important characteristic of the experiment is reproduced: COSET lines are strongly enhanced beyond the threshold for inelastic cotunneling connected to state $X$ [see arrows in Fig. 3(b)].

The appearance of a set of harmonic COSET lines at high bias can be explained by a subtle interplay of electronic and vibrational excitations, with two coexisting paths. Figure 3(a) illustrates these two paths: panels (1) and (2) for one and panels (1) and ( $\left.2^{\prime}\right)$ for the other. For energies larger than the one of the inelastic cotunneling step at $V_{\mathrm{SD}} \simeq-4 \mathrm{mV}$ a process involving two stages of inelastic cotunneling becomes feasible: the first effectively excites the dot from state $A$ to state $X$ [panel (1)], the second results in a transition from state $X$ into one of the
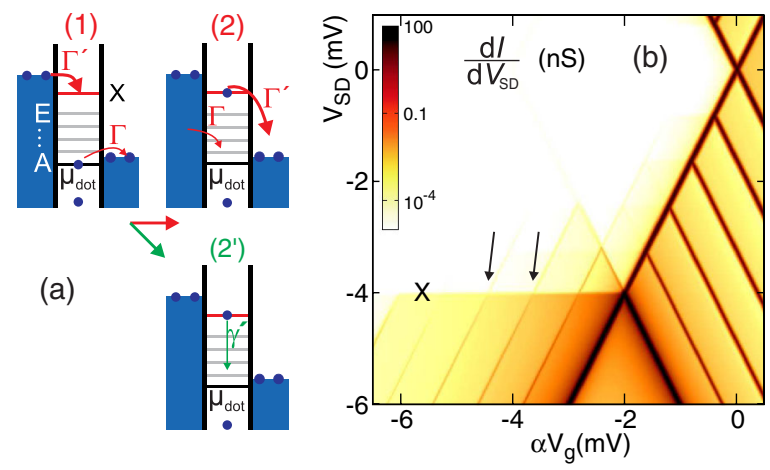

FIG. 3 (color online). Single quantum dot model reproducing the observed transport features. (a) Process and energy level scheme analogous to Fig. 2(f), leftmost panel, showing the lead Fermi levels, the accessible states within the quantum dot, and tunneling and relaxation rates relevant for pumping the vibration mode into nonequilibrium [17]. For a detailed description of the panels and the rates $\Gamma, \Gamma^{\prime}$, and $\gamma^{\prime}$, see the text. (b) Calculated differential conductance as function of $V_{g}$ and $V_{\mathrm{SD}}$, for the following parameters: vibrational level spacing $\hbar \omega=$ $810 \mu \mathrm{eV}, \quad k T=8.6 \mu \mathrm{eV}=10 \hbar \Gamma^{\prime}=10^{3} \hbar \Gamma=10^{4} \hbar \gamma^{\prime}=$ $10^{4} \hbar \gamma$. The tunnel couplings were chosen smaller than in the experiment to ensure a well-behaved perturbation expansion. $V_{g}$ is scaled with the gate conversion factor $\alpha=C_{g} / C$ (see, e.g., $[15,19])$. Arrows indicate the enhancement of COSET at high bias. states $B-E$ [panel (2)]. The rate for each such cotunnel process is $\propto \Gamma \Gamma^{\prime}$. Compared to the low-bias situation, this significantly enhances the population of the vibrational excited states $B-E$, and thereby the visibility of the vibrational COSET lines, since without access to state $X$, the limiting rates for COSET are proportional to $\Gamma^{2} \ll \Gamma \Gamma^{\prime}$. Thus, while COSET lines can in principle also be present at small bias $\left(\left|V_{\mathrm{SD}}\right|<4 \mathrm{mV}\right)$, beyond the inelastic cotunneling step they appear more pronounced: The strongly coupled excited state pumps the vibrational mode out of equilibrium, enhancing its population.

The second path that increases the occupation of the vibrationally excited states $B-E$ involves direct relaxation of state $X$ [panel $\left(2^{\prime}\right)$ in Fig. 3(a)] with a rate $\gamma^{\prime}$. As the precise value of $\gamma^{\prime}$ is unknown, we have verified that the qualitative result that the COSET lines are strongly enhanced beyond the inelastic cotunneling step connected to state $X$ persists for a large range of this parameter. An important further qualitative conclusion from the observation of COSET features is that relaxation from states $B-E$ into the two-hole ground state $A$ [rate $\gamma$ in Fig. 2(f)] in the experiment cannot be faster than tunneling out of the quantum dot $(\gamma \lesssim \Gamma)$.

So far we have assumed that the weakly gate-dependent feature $X$ in Fig. 2 corresponds to an electronic excited state. An alternative explanation invokes an electronic ground state of a second quantum dot forming within the nanotube, e.g., beneath one of the contact electrodes, as drawn in Fig. 4(a). Assuming this state has a much weaker gate coupling, we arrive at an equivalent mechanism for the nonequilibrium occupation of the vibrational mode. Local distortions and curving edges in the measurement of Figs. 1 and 2 may be taken as a hint of such a scenario, as has also been reported in Ref. [21].

Figure 4(b) illustrates the transport process for this "double quantum dot" case. The shielded additional small quantum dot displays a much weaker gate voltage dependence of the level energy. The line of enhanced differential conductance marked in Fig. 2 with $X$ now describes its alignment at the drain lead with the drain Fermi edge. The small quantum dot can then be occupied by a sequential tunneling process as sketched in Fig. 4(b). Inelastic cotunneling through the main dot may follow, leading to the occupation of an excited vibrational state. Although the
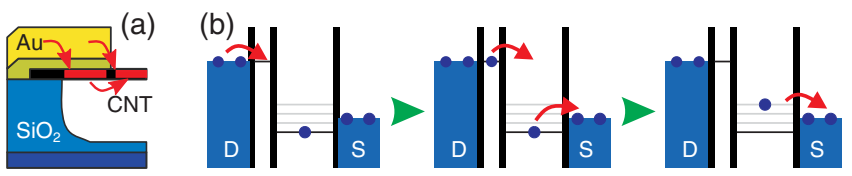

FIG. 4 (color online). Alternative mechanism for obtaining a vibrational nonequilibrium occupation. (a) Schematic side-view drawing detailing the possible formation of a small quantum dot beneath one of the metallic contact leads. (b) Transport processes for this "double quantum dot" case: additional cotunneling processes are enabled when the small quantum dot enters the energy window given by $V_{\mathrm{SD}}$ (see text). 
origin of, and tunneling processes associated with, state $X$ differ from the first scenario, the main effect is the same: state $X$ pumps the vibrational mode through consecutive tunneling events.

An intriguing feature of the measurement that has not been discussed so far is that the vibrational frequency in the $N_{h}=2$ charge state, as measured both from SET and COSET lines, is approximately twice as large as that in the $N_{h}=1$ charge state, seen as SET features only. This difference does not affect the pumping mechanism. It is possibly related to the fact that the observed quantum dot is in the few-carrier regime - in contrast to previous work on longitudinal phonon excitations in carbon nanotubes [6]. Whereas tension only affects the longitudinal mode via higher-order effects [6], the transition from, e.g., one to two trapped holes involves a distinct spatial redistribution of charge along the nanotube [12]. This strongly affects the electrostatic force distribution, which makes variations of vibration mode energies and mode shapes likely. As shown in Ref. [6], the coupling to the lowest symmetric vibrational mode of the nanotube may vanish for a charge distribution localized at the center of the nanotube. In general one may thus expect that the excitation of different modes depends strongly on the charge state, as we observe in the experiment.

Finally, the experimental observation of vibrational COSET resonances allows one to establish a lower boundary for the quality factor of the longitudinal mechanical mode [2], independent of the detailed pumping mechanism. The tunnel current at the edge of the low-bias $\mathrm{CB}$ region $\left(\left|V_{\mathrm{SD}}\right|<4 \mathrm{mV}\right), I \simeq 1 \mathrm{nA}$, provides an approximation for the SET tunneling rate $\Gamma$. For COSET to be visible, the lifetime of vibrational excitations must be larger than the corresponding time scale $\tau \simeq 0.16$ ns. With the vibrational energy $\hbar \omega=810 \mu \mathrm{eV}$, one obtains $Q \gtrsim$ $\tau \times \omega / 2 \pi=31$. Since we observe several sidebands, a higher value for $Q$ is more likely than this lower boundary, depending on the specific relaxation processes.

In conclusion, we have observed a pattern of equidistant, gate-dependent vibrational excitations in the Coulombblockade regime of a suspended carbon nanotube quantum dot. The appearance of the excitations is explained in the context of cotunnel-assisted sequential electron tunneling via phonon absorption processes. Interestingly, the absorption sidebands of the quantized phonon mode are only visible above a finite bias voltage threshold. Two models for the enhancement of the nonequilibrium distribution of the mechanical mode are discussed which demonstrate that the mode can be "pumped" either by an electronic excitation of the nanotube or by a ground state of another small quantum dot within the same nanotube. The pumping mechanism offers a perspective for electric control of quantized mechanical motion in nanoscale transistors by employing interplay with electronic degrees of freedom.
The authors would like to thank Y. Blanter and M. Poot for insightful discussions, and B. Otte and $\mathrm{H}$. Pathangi for experimental help. Financial support by the Dutch organization for Fundamental Research on Matter (FOM), the NWO VICI program, NanoNed, DFG SPP-1243, the Helmholtz Foundation, and the FZ-Jülich (IFMIT) is acknowledged.

*andreas.huettel@physik.uni-regensburg.de

Present address: Institute for Experimental and Applied Physics, University of Regensburg, 93040 Regensburg, Germany.

[1] S. Braig and K. Flensberg, Phys. Rev. B 68, 205324 (2003).

[2] M. C. Lüffe, J. Koch, and F. von Oppen, Phys. Rev. B 77, 125306 (2008).

[3] V. Sazonova, Y. Yaish, H. Ustunel, D. Roundy, T. A. Arias, and P. L. McEuen, Nature (London) 431, 284 (2004).

[4] B. Witkamp, M. Poot, and H. S. J. van der Zant, Nano Lett. 6, 2904 (2006).

[5] B. J. LeRoy, S. G. Lemay, J. Kong, and C. Dekker, Nature (London) 432, 371 (2004).

[6] S. Sapmaz, P. Jarillo-Herrero, Y. M. Blanter, C. Dekker, and H. S. J. van der Zant, Phys. Rev. Lett. 96, 026801 (2006).

[7] H. Park, J. Park, A. K. L. Lim, E. H. Anderson, A. P. Alivisatos, and P.L. McEuen, Nature (London) 407, 57 (2000).

[8] R. H. M. Smit, Y. Noat, C. Untiedt, N. D. Lang, M. C. van Hemert, and J. M. van Ruitenbeek, Nature (London) 419, 906 (2002).

[9] R. Leturcq et al., Nature Phys. 5, 327 (2009).

[10] M. Bockrath et al., Science 275, 1922 (1997).

[11] S. J. Tans et al., Nature (London) 386, 474 (1997).

[12] V. V. Deshpande and M. Bockrath, Nature Phys. 4, 314 (2008).

[13] F. Kuemmeth, S. Ilani, D. C. Ralph, and P. L. McEuen, Nature (London) 452, 448 (2008).

[14] P. Jarillo-Herrero, S. Sapmaz, C. Dekker, L. P. Kouwenhoven, and H.S.J. van der Zant, Nature (London) 429, 389 (2004).

[15] A. K. Hüttel, M. Poot, B. Witkamp, and H. S. J. van der Zant, New J. Phys. 10, 095003 (2008).

[16] J. Kong, H. T. Soh, A. M. Cassell, C. F. Quate, and H. Dai, Nature (London) 395, 878 (1998).

[17] See EPAPS Document No. E-PRLTAO-102-033924 for additional plots. For more information on EPAPS, see http://www.aip.org/pubservs/epaps.html.

[18] No clear features can be identified in the data at the positions expected for lines 4 and 10. This may, e.g., be due to electronic transitions shadowing the vibrational effects in transport.

[19] R. Schleser et al., Phys. Rev. Lett. 94, 206805 (2005).

[20] M. Leijnse and M. R. Wegewijs, Phys. Rev. B 78, 235424 (2008).

[21] K. Grove-Rasmussen, H. I. Jørgensen, T. Hayashi, P. E. Lindelof, and T. Fujisawa, Nano Lett. 8, 1055 (2008). 